\title{
Landau theory of the phase transitions in half-doped manganites: Interplay of magnetic, charge, and structural order
}

\author{
Fan Zhong \\ Department of Physics, University of Hong Kong, Hong Kong, People's Republic of China \\ and Department of Physics, Zhongshan University, Guangzhou 510275, People's Republic of China \\ Z. D. Wang* \\ Department of Physics, University of Hong Kong, Hong Kong, People's Republic of China
}

(Received 14 July 1999)

\begin{abstract}
The order parameters of the magnetic, charge, and structural orders at half-doped manganites are identified. A corresponding Landau theory of the phase transitions is formulated. Many structural and thermodynamical behaviors are accounted for and clarified within the framework. In particular, the theory provides a unified picture for the scenario of the phase transitions and their nature with respect to the variation of the tolerance factor of the manganites. It also accounts for the origin of the incommensurate nature of the orbital order and its subsequently accompanying antiferromagnetic order.
\end{abstract}

The discovery of "colossal" magnetoresistance has stimulated a renaissance of interest in doped rare-earth manganese. ${ }^{1,2}$ Intensive investigation has revealed a diversity of phenomena due to the complex interplay among magnetic, charge, orbital, and structural orders. A particular relevant issue is the competition between magnetic and charge orders in half-doped manganites. It poses a great challenge to theorists upon how to deal with the strong correlation in models with magnetic, orbital, and lattice degrees of freedom. Here we formulate a Landau theory of phase transitions based on the symmetry of the system in an attempt to understand a variety of sometimes controversial structural and thermodynamical behaviors. Although it may be argued to be only a mean-field theory, which is incorrect at critical points, the structural information, among others it affords is robust. And it is the order parameters that exhibit singularity at the critical points.

The most prominent charge ordered $(\mathrm{CO})$ behavior in perovskite manganites concerns with those doped at 0.5 . These systems exhibit several classes depending on the tolerance factor of the resultant structure (see Fig. 1). For $\mathrm{La}_{0.5} \mathrm{Sr}_{0.5} \mathrm{MnO}_{3}$ with small distortions, which we classify as class I hereafter though no charge order appears (neither does class II below), a paramagnetic (PM) to ferromagnetic (FM) transition occurs at $T_{C} \sim 360 \mathrm{~K}$. When La is replaced by a smaller ion $\mathrm{Nd}, T_{C}$ decreases with the tolerance factor. At $40 \%$ of $\mathrm{Nd}$ or so, an intermediately distorted class II shows up in which the FM phase transforms at a lower temperature $T_{A F M}$ to a metallic $A$-type antiferromagnetic (AFM) state. ${ }^{3,4}$ As more La is replaced by $\mathrm{Nd}^{4}{ }^{4}$ a new class III sets in that displays a CO CE-type AFM state below $T_{C O} \cdot{ }^{1}$ Similar behavior has been reported in $\mathrm{La}_{0.5} \mathrm{Ca}_{0.5} \mathrm{MnO}_{3}{ }^{5,6}$ $\mathrm{Pr}_{0.5} \mathrm{Sr}_{0.5} \mathrm{MnO}_{3}$ is somehow special. It was initially reported to be $\mathrm{CO},{ }^{7}$ but later only $A$-type AFM order was found. ${ }^{3}$ However, comparing the transport behavior of $\mathrm{Pr}_{0.5} \mathrm{Sr}_{0.5} \mathrm{MnO}_{3}$ with $\left(\mathrm{La}_{z} \mathrm{Nd}_{1-z}\right)_{0.5} \mathrm{Sr}_{0.5} \mathrm{MnO}_{3}$ of $0<z \leqslant 0.4$, one finds similar behavior. Only the latter's resistivity levels off to a presumed metallic state slightly slowly after a jump at $T_{C O}$ or $T_{A F M}$. So the boundary between the $\mathrm{CO}$ and $A$-type AFM states seems to be not so clear-cut: there may be a transition from the $\mathrm{CO}$ state to the $A$-type AFM state. For the most distorted class IV such as $\operatorname{Pr}_{0.5} \mathrm{Ca}_{0.5} \mathrm{MnO}_{3}$ and $\mathrm{Nd}_{0.5} \mathrm{Ca}_{0.5} \mathrm{MnO}_{3}$, no $\mathrm{FM}$ order appears. ${ }^{8}$ The PM phase changes directly into a $\mathrm{CO}$ state below $T_{C O}$ and then global AFM ordering shows up at a lower temperature $(\sim 150 \mathrm{~K})$. The CO tendency in class IV even extends to lower doping, although with a pseudo-CE-type structure due to the excess electrons. $^{8,9}$

The PM to FM transition is continuous. All the other transitions shown in Fig. 1 are of first order with hysteresis. The most striking feature of the $\mathrm{CO}$ state is that it can be melted by an external magnetic field, ${ }^{10}$ pressure ${ }^{4}$ or electric field ${ }^{11}$ or even by $\mathrm{x}$-ray ${ }^{12}$ or light irradiations ${ }^{13}$ into a FM state, indicating the competition between them. The required magnetic field to melt the $\mathrm{CO}$ state in class IV is almost twice as large as that in class III. ${ }^{14,15}$ In this paper, we shall concentrate on the peculiar phenomena associated with $\mathrm{CO}$. The lattice structure of interest is orthorhombic with a space group Pnma (see Fig. 1 inset), which is well characterized and the most common in doped manganites. There are some

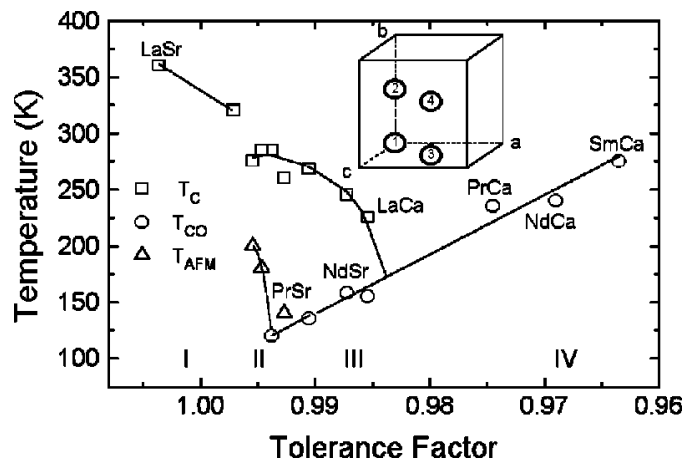

FIG. 1. Experimental phase diagram of half-doped manganites. Lines are only a guide for eyes. Sources of the data are given in the text. The inset displays the elementary unit cell of Pnma and the numbering of the four Mn ions. 
scatters in the reported structures. This is understandable because of the small distortion of the perovskite structure, which is also sensitive to the preparation conditions. We develop below a Landau theory of the phase transitions in these systems and show that many structural and thermodynamical behaviors are closely correlated within the framework. In particular, besides the magnetic, charge, and structural/ orbital pattern, primary features are a simple picture that unifies the three classes along with the nature of the transitions involved and the origin of the incommensurability of the orbital order, CE-type AFM order and the melting of $\mathrm{CO}$ state.

We start with the magnetic transition. A FM phase transition is associated with a wave vector at $\mathbf{k}=\mathbf{0}$ or the $\Gamma$ point. On the other hand, the CE-type AFM structure is described by $\mathbf{k}=\left(00 \frac{1}{2}\right)$ and $\mathbf{k}=\left(\frac{1}{2} 0 \frac{1}{2}\right)$ for Mn ions at positions 1,2 and 3,4 , respectively. In $\mathrm{La}_{0.5} \mathrm{Ca}_{0.5} \mathrm{MnO}_{3}$, the FM to AFM transition is found to be accompanying the incommensurate (IC) to (nearly) commensurate orbital ordering transition. ${ }^{5}$ In class IV, the CE-type AFM order can appear separate from the charge and structural orders, which produce a natural configuration for the CE-type AFM structure (see below). Thus the AFM order is regarded as originating from the charge and orbital orders. For simplicity, we leave it toward the end of the paper.

The PM to FM transition is described by an order parameter $M$ representing the average magnetization over the system. As pointed out in our previous work, ${ }^{16}$ all the irreducible representations (IR's) at $\mathbf{k}=\mathbf{0}$ of Pnma are one dimensional. So we choose $M$ as a scalar representing the component that carries the IR $\tau^{5}$ responsible for the transition. ${ }^{17}$ Although it may couple to other magnetic configurations such as $A$-type or $C$-type AFM orders of the same IR, we can eliminate such modes if present and write the free energy simply as

$$
F_{M}=\frac{1}{2} a_{1} M^{2}+\frac{1}{4} b_{1} M^{4}
$$

with $a_{1}=a_{10}\left(T-T_{1}\right)$, and $a_{10}$ and $b_{1}$ depending only weakly on the temperature $T$. Equation (1) describes a continuous phase transition at $T=T_{1}$ with $M=\sqrt{-a_{1} / b_{1}}$.

Next we move on to the $\mathrm{CO}$ phase transition. A prominent feature that indicates the existence of the $\mathrm{CO}$ state is the appearance of the superlattice diffraction spots characterized by a wave vector $\mathbf{k}=\left(\frac{1}{2} 00\right)$ or the $X$ point in the Pnma setting, which is adhered to throughout this paper. However, such spots associate more with lattice modulations than with the charge order that is characterized by $\mathbf{k}=(100)$ or $\Gamma$. Direct charge and orbital orders are first detected by $\mathrm{x}$-ray resonant scattering techniques in doped $\mathrm{La}_{2} \mathrm{SrMnO}_{4} \cdot{ }^{18}$ So to describe the transition, we need two order parameters.

On the one hand, the charge order is characterized by an order parameter $C=\xi_{1}+\xi_{2}-\xi_{3}-\xi_{4}$ with $\xi_{i}$ the occupancy possibility of site $i$ (Fig. 1 inset). $C$ is maximized if $\xi_{1}=\xi_{2}$ $=-\xi_{3}=-\xi_{4}$, and so a nonzero $C$ produces the observed $\mathrm{CO}$ pattern. By considering the permutations of the four sites under the symmetry operations of the Pnma group, it can be shown that $C$ transforms also as the IR $\tau^{5}$ of Pnma at $\Gamma$. Accordingly, the free energy of the $\mathrm{CO}$ transition is given by

$$
F_{C}=\frac{1}{2} a_{2} C^{2}+\frac{1}{4} b_{2} C^{4},
$$

where $a_{2}=a_{20}\left(T-T_{2}\right)$, and $a_{20}$ and $b_{2}$ are constants. Below $T_{2}$ charge order appears and the symmetry of the structure is lowered to $P 2_{1} / \mathrm{m}$. ${ }^{19}$

On the other hand, the structural modulation at $\mathbf{k}$ $=\left(\frac{1}{2} 00\right)$ is described by one of the two IR's of the wave vector, namely, $X_{1}$ and $X_{2}$, which are both two dimensional. ${ }^{17}$ Accordingly, the structural transition is characterized by a two-dimensional order parameter $\left(\eta_{1}, \eta_{2}\right)$. The physical meaning of the order parameter may be the displacement of the $\mathrm{Mn}^{4+} \mathrm{O}_{6}$ octahedra as modeled by Radaelli and co-workers to account for the diffraction patterns. ${ }^{6}$ It can be shown that $\eta_{2}$ may represent an arbitrary linear combination of the $x$ and $z$ components and $\eta_{1}$ the $y$ component of such displacements. Such a displacement pattern is consistent respectively with an orbital configuration of $d_{3 x^{2}-r^{2}}$ and $d_{x^{2}-y^{2}}$, which, when propagated half a period to $X$, switch to $d_{3 z^{2}-r^{2}}$ and $d_{y^{2}-z^{2}}$, respectively. It may be possible to choose alternatively an orbital basis such as $d_{3 x^{2}-r^{2}}$ and $d_{y^{2}-z^{2}}$ and then represent the order parameter as the long-range order of a certain orbital, which assumes a certain angle in the orbital space. We just note in passing that a certain displacement pattern corresponds to some orbital order.

Notice that the frequently observed $P 2_{1} / m$ symmetry can only arise from the IR $X_{1} \cdot{ }^{19}$ Therefore, the free energy for this structural transition is

$$
\begin{aligned}
F_{\eta}= & \frac{1}{2} a_{3}\left(\eta_{1}^{2}+\eta_{2}^{2}\right)+\frac{1}{4} b_{3}\left(\eta_{1}^{4}+\eta_{2}^{4}\right)+\frac{1}{4} d\left(\eta_{1}^{2}+\eta_{2}^{2}\right)^{2} \\
& +\kappa\left(\eta_{1} \frac{\partial \eta_{2}}{\partial x}-\eta_{2} \frac{\partial \eta_{1}}{\partial x}\right)+\frac{\sigma}{2}\left[\left(\nabla \eta_{1}\right)^{2}+\left(\nabla \eta_{2}\right)^{2}\right],
\end{aligned}
$$

where again $a_{3}=a_{30}\left(T-T_{3}\right)$, and $a_{30}, b_{3}, d, \kappa$, and $\sigma$ are constants.

A peculiar feature of Eq. (3) is the appearance of the Lifshitz invariant (the $\kappa$ term), which frequently leads to IC modulations. ${ }^{20,21}$ Many characteristic features of an IC transition has been observed in the $\mathrm{CO}$ and structural transition in manganites. ${ }^{6,22}$ Therefore, the IC nature of the structural/ orbital (but not charge) order has its origin in the Lifshitz invariant of the $X$ point. Nevertheless, we shall neglect this IC feature of the structural modulation below for simplicity and focus on its interplay with the charge and magnetic orders. This is partly justified by the fact that commensurate structure is also frequently observed in the same experiments that display the reverse. ${ }^{6}$ In this case, Eq. (3) then exhibits two possible phases below $T_{3}$. One has only one nonzero component equal to $\pm \sqrt{-a_{3} /\left(b_{3}+d\right)}$ and so its symmetry is $P 2_{1} / m^{19}$ if $b_{3}+d>0$ and $b_{3}<0$. The other satisfies $\eta_{1}$ $= \pm \eta_{2}= \pm \sqrt{-a_{3} /\left(b_{3}+2 d\right)}$ and belongs to Pm symmetry ${ }^{19}$ when $b_{3}+2 d>0$ and $b_{3}>0$.

Coupling of the charge to the structural degrees of freedom can be readily found by noting that $\eta_{1}^{2}-\eta_{2}^{2}$ transforms as the same IR as $C$, and so the simplest coupling between them is 


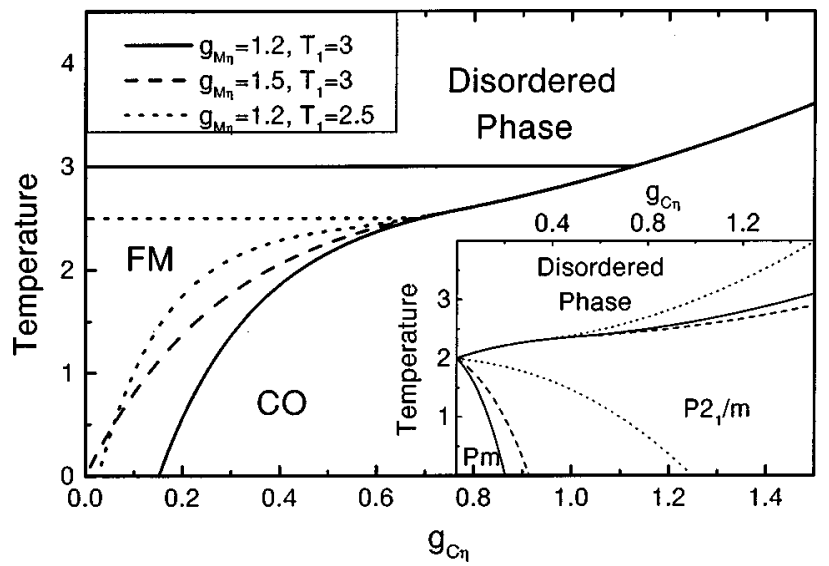

FIG. 2. Theoretical phase diagram of the coupled magnetic, charge, and structural transitions. The parameters are $a_{10}=a_{20}$ $=a_{30}=b_{1}=b_{2}=b_{3}=1, T_{1}=3, T_{2}=2.5, T_{3}=2$, and $g_{M \eta}=1.2$ (solid lines). The dashed line corresponds to $g_{M \eta}=1.5$, while the dotted lines to $T_{1}=2.5$. Inset: phase diagram of the chargestructural transition. The dashed lines represent $b_{3}=2$, while the dotted ones $d=-0.3$.

$$
F_{C \eta}=g_{C \eta} C\left(\eta_{1}^{2}-\eta_{2}^{2}\right),
$$

where $g_{C \eta}$ is a measure of the coupling. Assume $g_{C \eta}$ and $C$ are positive without loss of generality. It is transparent then that the coupling will favor the ordering of $\eta_{2}$ once the charge is ordered, since the transition point for $\eta_{2}$ is now elevated to $a_{3}-2 g_{C \eta} C$, while that for $\eta_{1}$ lowered to $a_{3}$ $+2 g_{C \eta} C$. This explains the reason for the often observed $P 2_{1} / m$ symmetry. ${ }^{6}$ Possibility for ordering of both $\eta_{1}$ and $\eta_{2}$ still exists, which may account for the absence of the $2_{1}$ screw axis in $\mathrm{Sm}_{0.5} \mathrm{Ca}_{0.5} \mathrm{MnO}_{3}$, which should then be of $P m$ symmetry, though it was preferred to be $P 2 \mathrm{~mm}$ or $P m m m .{ }^{23}$ This scenario is confirmed from the phase diagram illustrated in the inset of Fig. 2. It is seen that as $b_{3}$ increases, the boundary of the $P m$ phase moves to the right, reducing the region of the $P 2_{1} / m$ phase. If $b_{3}<0$, only the $P m$ phase exists, while for $d<0$, the $P m$ phase extends far to the right. Note that a large $b_{3}$ or small $d$ means a large "lock-in" term [the $b_{3}$ term in Eq. (3)] that tends to suppress the incommensurability. ${ }^{21}$ This seems to be consistent with the observation that more distorted systems such as Sm and Gd tend to be more stoichiometric and so commensurate. ${ }^{23} \mathrm{We}$ remark that the transition from $P 2_{1} / m$ to $P m$ phase may accompany or be hidden by the IC to commensurate transition and PM to CE-type AFM transition.

Since $M$ changes sign by time reversal, the only possible couplings of the magnetic to the charge and structural transition are biquadratic, i.e.,

$$
F_{M C \eta}=\frac{1}{2} g_{M \eta} M^{2}\left(\eta_{1}^{2}+\eta_{2}^{2}\right)+\frac{1}{2} g_{M C} C^{2} M^{2}
$$

where both coupling coefficients are positive due to the competing orders.

Equations (1)-(5) constitute our theory of the magnetic and charge and structural or orbital transitions. Instead of going into detailed estimations of the various coefficients in the model, we are content here with global features that are believed to be relevant to the parameter regime of real ma-

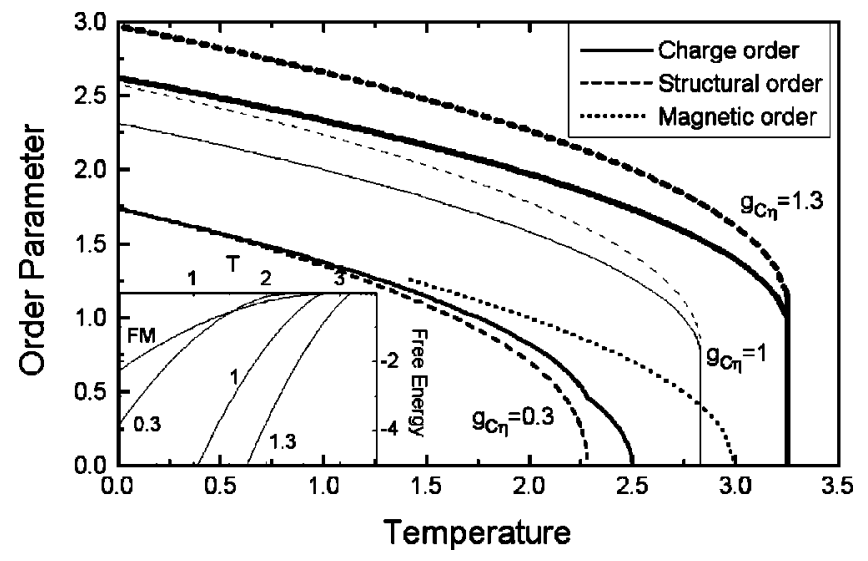

FIG. 3. Order parameter vs temperature $T$. Thick lines correspond to $g_{C \eta}=1.3$, while thin and mediate lines $g_{C \eta}=1$ and $g_{C \eta}$ $=0.3$, respectively. The inset shows the corresponding free energy from bottom. The upper curve is for the pure FM phase [Eq. (1)]. Other parameters are the same as the solid lines in Fig. 2. Overlaps in $g_{C \eta}=0.3$ imply metastable region.

terials. To this end, we study of a simplified version of the theory, in which we have taken $\eta_{1}=0$, i.e., disregarded the possibility of the $P m$ symmetry, relabeled $b_{3}+d$ as $b_{3}$, and neglected the biquadratic coupling between the charge and magnetism, since their coupling to the structural order results in a lower-order $C M^{2}$-type coupling. This implies that longrange charge order always accompanies with structural order.

Figure 2 displays a generic phase diagram for several sets of the parameters in arbitrary units. It shows the strong coupling case $g_{M \eta}^{2}>b_{1} b_{3}$ between magnetism and lattice so that no mix magnetic and charge orders appears. It can be seen that as $g_{C \eta}$ increases, the sequence of phase transitions changes from a $\mathrm{PM} \rightarrow \mathrm{FM}$ to a $\mathrm{PM} \rightarrow \mathrm{FM} \rightarrow \mathrm{CO}$ then to a $\mathrm{PM} \rightarrow \mathrm{CO}$ phase directly as $T$ is lowered. The real situations as in Fig. 1 are, of course, not simply only a variation of $g_{C \eta}$, but Fig. 2 exhibits in a simple way the relevance of the theory. Figure 3 shows the variation of the order parameters for three different $g_{C \eta}$. It is seen that the transition to $\mathrm{CO}$ phase is discontinuous. The variations of $C$ and $\eta$ are similar, but their coupling is not necessarily linear. ${ }^{24}$ Note that the $\mathrm{CO}$ and the structural transitions are so strongly coupled that they take place at a single transition temperature. Although the $\mathrm{CO}$ can still appear preceding the structural order when $g_{C \eta}<1$, the reverse is not true, namely, $\eta=0$ if $C$ $=0$. Therefore the structural or orbital order is driven by the charge order. The inset plots the corresponding free energy vs temperature, showing that the larger the $g_{C \eta}$, the lower the free energy, and so the bigger the magnetic field that lowers the upper curve [Eq. (1)] by $H M$ to melt the CO state, being in agreement with experiments. This indicates that the magnetic field acts in melting the $\mathrm{CO}$ state more than the AFM state. An evidence for this is that above the CE-type AFM ordering temperature, no AFM for the field to melt. This also justifies the separate treatment of the AFM order.

Finally we discuss briefly the transition to the CE-type AFM order. Noting that in $\mathrm{La}_{0.5} \mathrm{Ca}_{0.5} \mathrm{MnO}_{3}$ with the standard CE-type state, the magnetic moments lie in the $a-c$ plane, ${ }^{6}$ we may choose the $x$ and $z$ components of the AFM vectors $\mathbf{L}_{1}=\boldsymbol{\mu}_{1}-\boldsymbol{\mu}_{2}$ and $\mathbf{L}_{2}=\boldsymbol{\mu}_{3}-\boldsymbol{\mu}_{4}$ as order parameters, since they transform as the two components of the two- 
dimensional IR's $\tau^{1}$ and $\tau^{1}$ combining with its complex conjugate $\tau^{3}$ associated respectively with the wave vectors (00 $\left.\frac{1}{2}\right)$ and $\left(\frac{1}{2} 0 \frac{1}{2}\right),{ }^{17}$ where $\boldsymbol{\mu}_{i}$ is the magnetic moment of ion $i$. The two IR's rather than one makes the transition discontinuous. ${ }^{21}$ Both IR's give a magnetic symmetry of $P_{b} 2_{1} / m$ and a structural one of $P 2_{1} / m$ that is identical with the $\mathrm{CO}$ and structural transitions. ${ }^{19}$ The compatibility of the orbital and magnetic patterns implies an enhancement of both transitions and can be described by a coupling $\left(\eta_{1}^{2}\right.$ $\left.+\eta_{2}^{2}\right)\left(L_{1 \alpha} L_{1 \beta}+L_{2 \alpha} L_{2 \beta}\right)$ with a negative coupling constant, where $\alpha$ and $\beta$ denote $x$ or $z$. As a result, ordering of one kind of the orders enhances the other, leading, for instance, to the accompanying of the AFM order with the IC to commensurate orbital order ${ }^{5,22}$ as the AFM order promotes the contribution of the lock-in term, and to the increasing of resonant $\mathrm{x}$-ray scattering intensity of charge and orbital orders as AFM ordering. ${ }^{24}$

In conclusion, we have developed a Landau theory for the coupled phase transitions in half-doped manganites through the identification of the order parameters for the FM, CEtype AFM, CO and structural or orbital orders. The theory provides a unified picture for the scenario of the phase transitions and their nature with respect to the variation of the tolerance factor of the manganites via the symmetry-adapted coupling among the degrees of freedom. Many peculiar phenomena of half-doped manganites result from the interplay between the FM or A-type AFM and CO states, the CE-type AFM sets in only as a secondary factor. So an applied magnetic field primarily melts the $\mathrm{CO}$ state. The theory also accounts for the origin of the IC nature of the orbital order and its subsequently accompanying AFM order. As a phenomenological theory, it can make direct contact with the experimental results especially the symmetry of the involved structures that sensitively influence the transport behavior in manganites. Experimental clarifications are desirable of the symmetry of the $\mathrm{CO}$ state and its relation to the oxygen stoichiometry and commensurablity, possible structural transition from $P 2_{1} / m$ to $P m$, and the relation between $A$-type AFM and $\mathrm{CO}$ states.

This work was supported by a URC fund and a CRCG grant at the University of Hong Kong.
*Electronic address: zwang@hkucc.hku.hk

${ }^{1}$ E.O. Wollan and W.C. Koehler, Phys. Rev. 100, 545 (1955).

${ }^{2}$ R.M. Kusters et al., Physica B 155, 141 (1989); K. Chahara et al., Appl. Phys. Lett. 63, 1990 (1993); R. von Helmolt et al., Phys. Rev. Lett. 71, 2331 (1993); S. Jin et al., Science 264, 413 (1994); F. Zhong, J.M. Dong, and Z.D. Wang, Phys. Rev. B 58, 15310 (1998); S. Q. Shen and Z. D. Wang, Phys. Rev. B 58, R8877 (1998); ibid. 59, 14484 (1999).

${ }^{3}$ H. Kawano et al., Phys. Rev. Lett. 78, 4253 (1997).

${ }^{4}$ Y. Moritomo et al., Phys. Rev. B 55, 7549 (1997); T. Akimoto et al., ibid. 57, R5594 (1998).

${ }^{5}$ P.G. Radaelli et al., Phys. Rev. Lett. 75, 4488 (1995); C.H. Chen and S.W. Cheong, ibid. 76, 4042 (1996).

${ }^{6}$ P.G. Radaelli et al., Phys. Rev. B 55, 3015 (1997).

${ }^{7}$ K. Knizek et al., J. Solid State Chem. 100, 292 (1992); Y. Tomioka et al., Phys. Rev. Lett. 74, 5108 (1995).

${ }^{8}$ Z. Jirak et al., J. Magn. Magn. Mater. 53, 153 (1985); Y. Tomioka et al., Phys. Rev. B 53, R1689 (1996); M. Tokunaga et al., ibid. 57, 5259 (1998).

${ }^{9}$ H. Yoshizawa et al., Phys. Rev. B 52, R13 145 (1995); D.E. Cox et al., ibid. 57, 3305 (1998).
${ }^{10}$ H. Kuwahara et al., Science 270, 961 (1995).

${ }^{11}$ A. Asamitsu et al., Nature (London) 388, 50 (1997).

${ }^{12}$ V. Kiryukhin et al., Nature (London) 386, 813 (1997).

${ }^{13}$ K. Miyano et al., Phys. Rev. Lett. 78, 4257 (1997).

${ }^{14}$ M. Tokunaga et al., Phys. Rev. B 57, 5259 (1998).

${ }^{15}$ G. Xiao et al., J. Appl. Phys. 81, 5324 (1997).

${ }^{16}$ F. Zhong and Z.D. Wang, Phys. Rev. B 60, 11883 (1999).

${ }^{17}$ O. V. Kovalev, Representations of the Crystallographic Space Groups, edited by H. T. Stokes and D. M. Hatch (Gordon and Breach, Yverson, 1993).

${ }^{18}$ Y. Murakami et al., Phys. Rev. Lett. 80, 1932 (1998).

${ }^{19}$ H. T. Stokes and D. M. Hatch, Isotropy Subgroups of the 230 Crystallographic Space Groups (World Scientific, Singapore, 1988).

${ }^{20}$ E.M. Lifshitz, Zh. Eksp. Teor. Fiz. 11, 255 (1941).

${ }^{21}$ J. C. Tolédano and P. Tolédano, The Landau Theory of Phase Transitions (World Scientific, Singapore, 1987).

${ }^{22}$ S. Mori et al., Phys. Rev. B 59, 13573 (1999).

${ }^{23}$ A. Barnabe et al., J. Appl. Phys. 84, 5506 (1998); J. Mater. Chem. 8, 1405 (1998).

${ }^{24}$ M.v. Zimmermann et al., Phys. Rev. Lett. 83, 4872 (1999). 